\title{
Inflación importada vía tipos de cambio y su aplicación al caso colombiano mediante el uso de modelos VEC para el periodo enero 2010 - diciembre 2018
}

Inflation imported via exchange rates and its application to the Colombian case through the use of VEC models for the period January 2010 - December 2018

Heivar Yesid Rodríguez Pinzón ${ }^{\mathrm{a}}$ heivarrodriguez@usantotomas.edu.co

Leidy Rincón ${ }^{\mathrm{c}}$ leidy.rincon@unillanos.edu.co
Stephania Agudelo Galindo ${ }^{\mathrm{b}}$

stephania.agudelo@usantotomas.edu.co

Katherine Montañez ${ }^{\mathrm{d}}$

katherine.montanez@unillanos.edu.co.

Resumen

La finalidad del presente documento es el análisis del efecto de las variables Tasa Representativa de Mercado (TRM) e Índice de Tasa de Cambio Real (TCR) en el Índice de Precios al Consumidor para Colombia (IPC), tomando como base el periodo comprendido entre el mes de enero 2010 y el mes de diciembre del año 2018. La estrategia de estimación se realizó mediante el modelo VEC (Vector de Corrección de Errores), el cuál arrojó evidencia que confirma un escenario de inflación importada en Colombia, comprobando la endogeneidad del proceso inflacionario.

Palabras clave: Inflación, Inflación importada, modelos VEC.

Abstract

The purpose of this paper is the analysis of the effects of variables Representative Market Rate (TRM) and Index of Real Exchange Rate (TCR) in the consumer Price Index (IPC) for Colombia, based on the period from January 2010 to December 2018. The estimation strategy was made using the VEC model (Vector Error Correction), which showed evidence that confirm an imported inflation scenery in Colombia, proving the endogeneity of national inflation.

a Magister en Ciencias Económicas. Docente Tiempo Completo. Facultad de Estadística. Universidad Santo Tomás. Dirección de Correspondencia: Carrera 7 No. 51 A 13 Piso 3. Facultad de Estadística.

b Profesional en Negocios Internacionales. Profesional en Economía. Facultad de Economía. Universidad

Santo Tomás.

c Estudiante de Economía. Programa de Economía. Universidad de Los Llanos

d Estudiante de Economía. Programa de Economía. Universidad de Los LLanos. 
Keywords: Inflation, imported inflation, VEC models.

\section{Introducción}

Los significativos efectos del fenómeno inflacionario en la coyuntura económica destacan la importancia de su respectivo seguimiento, pronóstico y posterior análisis. Lo anterior, en aras de posibilitar una percepción sobre su impacto en la estabilidad macroeconómica, revelando la información necesaria y facultando así a las autoridades monetarias para la toma de decisiones pertinentes. "El desarrollo y perfeccionamiento de un sistema de pronóstico de la inflación acompañó la decisión del Banco de la República de adoptar formalmente el esquema de inflación objetivo" (Urrutia, M., 2002). Mediante este modelo la Junta Directiva del Banco de la República determina una meta de inflación, la cual en niveles estables mantendrá el poder adquisitivo de la moneda nacional, incrementará la competitividad, promoverá crecimiento sostenible y será generador de confianza en el mercado.

Evidencia empírica demuestra que la oferta monetaria no es el único determinante de dicho nivel generalizado de precios. Fenómenos ambientales y/o dinámicas sociales que afectan los procesos de producción y comercio de alimentos, niveles de incertidumbre en la economía y mecanismos de indexación, son factores nacionales de gran influencia para el nivel de precios en la economía. Sin embargo, actualmente, los procesos que se desarrollan para la generación de políticas que cumplan con metas inflacionarias deben también tener en cuenta factores del ámbito internacional. Circunstancias como los niveles de crecimiento de los principales socios comerciales, fluctuaciones en el valor del dólar estadounidense, variaciones en los precios internacionales de bienes primarios, el desempeño económico de las grandes economías en América Latina, y la oscilante volatilidad del mercado bursátil mundial, son sucesos que provocan un desajuste en las expectativas de inflación.

Un factor de considerable relevancia, es la fluctuación en los precios de los bienes y servicios externos. Dicho efecto sobre la inflación interna se conoce como inflación importada y puede medirse para las economías en desarrollo y abiertas por tres caminos: el primero está ligado a la canasta de productos importados, en segundo lugar, la inflación interna de los países con los que se presenta un mayor intercambio comercial, específicamente mayor cantidad de importaciones, y en último lugar, mediante las variaciones que se presentan en la tasa de cambio.

El nombre que recibe el efecto de modificaciones en la TRM sobre la inflación interna, se conoce como coeficiente de traspaso. La magnitud de este depende de múltiples factores, entre ellos el nivel de volatilidad del tipo de cambio per se y el nivel de 
estabilidad de la inflación en la economía, importante para el valor de la elasticidad precio-demanda de los consumidores. El aumento generalizado de los precios implicará un cambio en precios relativos guiado hacia una disminución del ingreso real, de no darse un cambio equivalente en los ingresos. Rincón (2000) sostiene que los efectos de una variación en la tasa de cambio dependerán del grado de desalineamiento de la tasa de cambio real de su nivel de equilibrio. Esta afirmación señala una correlación entre el coeficiente de traspaso y las desviaciones de la TCR. Lo anterior supone que en un escenario donde la tasa de cambio real se encuentre en equilibrio, la alteración observada en el tipo de cambio será de igual magnitud en el nivel de los precios domésticos. Así mismo, cuando la tasa de cambio real está depreciada, un incremento en el tipo de cabio tendrá un mayor impacto en los precios domésticos, que conduzca al TCR de vuelta a su punto de equilibrio.

Dadas las presiones económicas, Colombia no es ajena a los coletazos de las economías externas. Lo anterior, abre el interrogante sobre el impacto en la inflación interna, y reitera el carácter imperioso de tener en cuenta en la formulación de políticas encaminadas a la inflación objetivo, el papel de los factores externos en los precios domésticos, con el fin de tener un mayor control sobre los efectos del fenómeno de inflación importada, especialmente al ser analizada por la defensa de la moneda vía tasa cambiaria, por los movimientos del Índice de Tasa de Cambio Real (TCR), o la variación de los precios del petróleo y su impacto en los precios domésticos. Fenómeno analizado en el documento de Rodríguez (2011).

Según González, Rincón \& Rodríguez (2008) "El supuesto de transmisión completa de la tasa de cambio sobre los precios tiene su origen en los modelos monetarios de la tasa de cambio, [...] en la ley de un solo precio, o su generalización, hipótesis de poder de paridad de compra". Teorías que plantean la correlación entre el tipo de cambio y los precios de un bien entre dos países. Este documento tiene como propósito mostrar un escenario de inflación importada para el caso colombiano, y exponer los impactos directos causados por una variación en las variables externas TCR y TRM, en el Índice de Precios al Consumidor colombiano (IPC). En aras de lo anterior, se presenta una revisión literaria sobre el fenómeno de inflación importada vía tipo de cambio nominal y tipo de cambio real, y su aplicación para el caso nacional en el periodo comprendido entre el mes de enero del año 2010 y el mes de diciembre del año 2018.

\section{Política Económica y su relación con la inflación y los tipos de cambio}

El principal objetivo de la política monetaria es preservar el valor y confianza de la moneda nacional, como también estabilizar la economía (Cárdenas M., 2013), ante este propósito, la meta inflacionaria se convierte en una meta preponderante para la estabilidad macroeconómica de la nación y el bienestar de la población. En este orden 
de ideas, entendiendo que el índice de precios al consumidor (IPC) "mide la evolución del costo promedio de una canasta de bienes y servicios representativa del consumo final de los hogares, expresado en relación con un período base" (Banco de la República, s.f), se reitera la fluctuación de este como gran determinante del ingreso real del consumidor nacional y del poder adquisitivo del peso colombiano. En lo que respecta a su definición como tal, el hecho que no haya un consenso en su formulación, y de las variables que deben estar implícitas en él, lleva a muchos economistas e investigadores económicos a dudar de la variación en el IPC y las medidas a implementar al respecto.

El régimen de tipo de cambio flexible bajo el cual se rige actualmente Colombia, facilita a las autoridades monetarias la adopción de medidas anti cíclicas en épocas de crisis con el fin de dinamizar la economía, es allí donde los sistemas de pronóstico adquieren gran relevancia. Tener la mayor certeza posible respecto al impacto de una fluctuación de la TRM en los precios domésticos, faculta a las autoridades monetarias para la toma de medidas pertinentes con el fin de contrarrestar dichos efectos, influyendo en la demanda agregada mediante políticas económicas que deben seguir un solo canal conductor, el cual debe pretender de forma coherente una mejora conjunta de múltiples ámbitos de la economía nacional, tales como la capacidad del aparato productivo nacional, la balanza comercial y el empleo. A partir de lo anterior, Urrutia (2004) afirma "Si la autoridad monetaria modificó su postura de política monetaria frente a los movimientos de la tasa de cambio, fue porque consideró que ellos comprometían el logro de las metas de inflación, o contribuían a la desviación del producto observado frente al producto potencial".

Sobre la política monetaria "se ha hecho recaer desde hace muchos años la responsabilidad principal por el control de la demanda agregada en las economías desarrolladas y en desarrollo. Pero ciertamente hay poca discusión y experiencia con respecto al rol de freno de la política fiscal" (Frenkel R., 2008). La política monetaria, el actor dominante del flujo de efectivo en la economía, mediante la tasa de interés, compra venta de divisas, operaciones de mercado abierto o TES, incide en el tipo de cambio nominal con el objetivo de cumplir metas inflacionarias y de balanza comercial. Sin embargo, se le atribuye un efecto transitorio debido a que su impacto en la oferta monetaria se encuentra mitigado después de determinado periodo de tiempo, ya que el tipo de cambio nominal y el nivel de precios llegan a una proporción equivalente a la inicial, consecuencia de la homogeneidad grado uno de estas variables respecto al dinero. Es por lo anterior, que la política fiscal se convierte en el elemento clave para mitigar presiones inflacionarias, ya que como afirman Rendón \& Ramírez (2005) "la influencia del gasto público sobre la balanza comercial presenta dos efectos; uno directo vía absorción y gasto transitorio y un efecto indirecto vía tasa de cambio real". Por consiguiente se obtiene un control de la inflación y un beneficio en la balanza comercial vía reducción de la absorción interna. 
"Después de estandarizar los bienes y servicios tanto nacionales como importados, expresados en la moneda local, se obtienen los precios relativos, indicadores que determinan los flujos del comercio internacional" (Krugman \& Obstfeld, 1994). Este patrón de precios, denominado índice de tasa de cambio real (TCR), está directamente influenciado por el nivel de precios nacional e internacional y el tipo de cambio nominal. Es uno de los indicadores de mayor relevancia en la economía, pues su valor manifiesta el nivel de competitividad de los precios nacionales en comparación con los extranjeros. Es por lo anterior que, estudiar el efecto del tipo de cambio, como el canal de transmisión de la política monetaria toma mayor relevancia.

El impacto de las políticas económicas sobre los tipos de cambio dependerá del grado de apertura de la economía; específicamente de la integración financiera del país con el exterior (o de la movilidad de capital). Según Mundell (1963) se identifica que si hay un grado alto de intercambio financiero con el resto del mundo, el tipo de cambio será razonable si se logra mantener la igualdad de la rentabilidad esperada entre activos domésticos y extranjeros con características similares, ambos denominados en la misma moneda.

\section{Inflación importada}

El fenómeno de inflación importada se presenta cuando se genera un alza en el nivel generalizado de precios de una economía, por factores externos al mercado nacional. Este efecto en los precios, fue definido por Frisch (1977) como "la transmisión directa de la inflación por medio de los bienes comercializados internacionalmente". Urrutia (2004) afirma que los efectos de la tasa de cambio sobre la inflación pueden ser temporales, y afectar la inflación de manera indirecta mediante su impacto en la demanda de bienes y servicios nacionales.

En el trabajo de Rincón, Lozano \& Ramos (2008) se presentan los canales de transferencia del efecto de los tipos de cambio en la inflación. Se expone el principal de ellos de tipo cambiario "el cual tiene origen en la entrada súbita de recursos externos que tienden a revaluar el tipo de cambio real, tanto por los recursos que efectivamente ingresan a la economía como por las expectativas que se generan. Este fenómeno se manifiesta finalmente en un aumento del consumo de bienes importados y en el deterioro la cuenta corriente de la balanza de pagos.

El segundo canal de transmisión macroeconómica proviene del efecto ingreso derivado del mejoramiento de los términos de intercambio, el cual se manifiesta en un aumento del consumo... Finalmente, el tercer canal de transmisión del choque petrolero es el fiscal, el cual inicialmente se manifiesta en un aumento de los ingresos públicos, sobre todo en aquellos países donde este tipo de rentas son apropiadas mayoritariamente por el Estado, como es el caso de Colombia. Es necesario anotar que a mediano plazo, las rentas extraordinarias provenientes de una bonanza no estimulan el ahorro público sino que, por el contrario, pueden generar mayor gasto de manera permanente." 
Efecto de los tipos de cambio sobre la inflación

El nivel de correlación entre el tipo de cambio nominal y la inflación ha sido un fenómeno altamente documentado, y presenta teorías representativas sobre la proporción que ambas deben mantener respecto a la otra. Solé, R (2012) cita a Ross, Westerfield \& Jaffe (2010) quienes indican que la Paridad del Poder de Compra (PPC) "señala que la variación en el tipo de cambio queda determinada por la diferencia en las tasas de inflación de los dos países", por consiguiente se afirma que la tasa de cambio nominal debe ajustarse según los niveles de precios para que ambas monedas mantengan sus poderes adquisitivos, ajuste determinado por el índice Big Mac.

La reacción de la inflación interna ante un cambio en el tipo de cambio nominal implica un nexo de causalidades en el que entran en juego múltiples factores aparte de la oferta monetaria o la sustitución de importaciones. En el traspaso vía tipo de cambio nominal, ante una situación de depreciación de la moneda nacional se presentan dos efectos: El efecto valor y el efecto volumen. Una consecuencia directa y a corto plazo de una disminución del valor de la moneda nacional, es una reducción del precio de las exportaciones para los compradores extranjeros, por consiguiente mayores ingresos en pesos para los exportadores nacionales, y un encarecimiento de las importaciones para los compradores nacionales. El segundo efecto, de volumen, cuyo impacto se evidencia en un lapso de tiempo mayor, se asocia a un incremento y disminución, de las exportaciones e importaciones respectivamente, determinado por el aparato productivo del país. En el efecto valor, el traspaso del alza en los costos a los consumidores finales, con un mayor énfasis en el encarecimiento de mercancías para los importadores, está supeditado a una serie de factores coyunturales, que precisan si las empresas deben modificar sus precios o alterar su margen de ganancia. Si en primer lugar, la depreciación de la moneda se considera una situación temporal, las empresas no incurrirán a los costos que abarca un cambio en sus precios, más aún si se encuentra en un escenario de economía en recesión o de inflación estable, donde los consumidores presentarían una elasticidad precio - demanda mucho mayor. Por lo anterior, la empresa recurrirá a una reducción de sus ganancias conforme la situación se estabiliza, a cambio de no perder participación en el mercado. En un escenario distinto, donde se conjetura una depreciación mucho más prolongada, las empresas preferirán un cambio en sus precios para no generar pérdidas al largo plazo.

\section{Metodología}




\section{Cointegración}

Engle \& Granger (1987), postulan que una combinación lineal de dos o más series no estacionarias puede llegar a ser estacionaria. A esta particularidad se le conoce como cointegración, y se presenta cuando las variables mantienen una relación estable a largo plazo y en el corto plazo, hay errores que se le atribuyen a una aleatoriedad en su comportamiento. La prueba de cointegración que se llevó a cabo fue bajo la metodología de Johansen \& Juselius (1990), los resultados de las pruebas de cointegración se aprecian en el anexo 1.

\section{Modelo VEC.}

El modelo VEC es uno de los modelos utilizados en el análisis de series de tiempo multivariados, su principal característica está en que las variables presentan cointegración, es decir, una relación de equilibrio en el largo plazo.

Teniendo en cuenta el trabajo de Lettal \& Ludvigson (2001), en el cual presentan las relaciones teóricas que con llevan a la relación de equilibrio de largo plazo entre las variables del modelo y contemplando el resultado de la prueba de Johansen \& Juselius (1990), se propone el uso de modelos VEC, los cuales han sido la expansión de los modelos VAR (Vector AutoRegressive), debido a que incluye las dinámicas de ajuste en el corto plazo, cuando se presenta un shock, presentando información acerca de la velocidad de ajuste hacia el equilibrio, por consiguiente el modelo VEC ofrece más información que el modelo VAR (Vector AutoRegressive) (Fernandez-Corugedo, 2003). Se recomienda para una explicación más detallada de la estructura teórica del modelo VEC el texto de Wei (2006).

Para ello el procedimiento será el modelamiento de las variables por pares (IPC - TRM y IPC - TCR), con el fin de mostrar que el fenómeno de inflación importada está presente en el caso Colombiano.

\section{Resultados}

Para cumplir con las condiciones mencionadas anteriormente en el ajuste del modelo VEC, se verificará el orden de integración, y se trabajará con series transformadas con la función logaritmo natural para facilitar su lectura y de ser necesario se efectuarán transformaciones para cumplir con los supuestos. En todas las variables consideradas se utilizó el periodo comprendido entre el mes de enero de 2006 hasta el mes de enero de 2016. Todas las pruebas y los modelos se leerán con un 95\% de confianza.

\section{A nálisis gráfico preliminar}

Se observa la Figura 1 la serie mensual del IPC total en Colombia, en ella se aprecia un comportamiento creciente, y dado la estacionalidad histórica de la serie, se incluirá la prueba de estacionalidad para determinar la existencia o no en el periodo estudiado. 


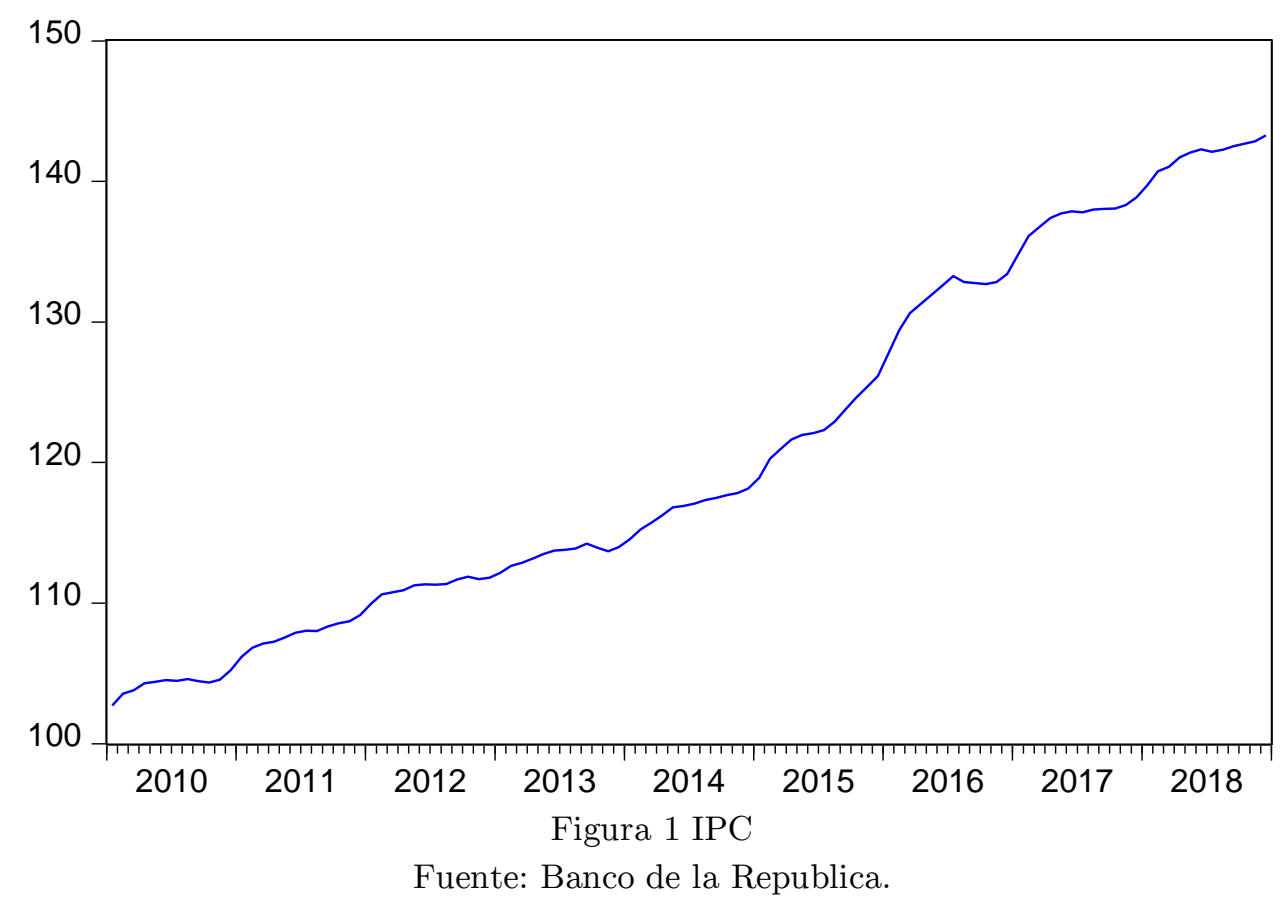

Para determinar la estacionalidad de la serie, se utilizó el proceso CENSUS X12 del paquete EVIEWS teniendo en cuenta el trabajo Kikut \& Ocampo (2005), donde se aprecia de forma detallada su uso. En la siguiente salida se presenta las pruebas de estacionalidad y la variable IPC_SA (SA corresponde a la variable sin el componente estacional: Seasonal Adjusted). Para una explicación más detallada del uso del proceso $\mathrm{X} 12$ y de las pruebas estadísticas que arroja el software, se recomienda el trabajo de Gallardo \& Rubio (2009).

Test for the presence of seasonality assuming stability.

\begin{tabular}{crrrrr} 
& Sum of & Dgrs.of & \multicolumn{2}{c}{ Mean } & \\
Squares & Freedom & Square & F-Value \\
Between months & 16.9122 & 11 & 1.53747 & $67.019^{* *}$ \\
Residual & 2.2023 & 96 & 0.02294 & \\
Total & 19.1145 & 107 & & \\
& & & &
\end{tabular}

**Seasonality present at the 0.1 per cent level.

Nonparametric Test for the Presence of Seasonality Assuming Stability

Kruskal-Wallis Degrees of Probability

Comunicaciones en Estadística Junio 2019, Vol. 12, No. 1 


$\begin{array}{ccc}\text { Statistic } & \text { Freedom } & \text { Level } \\ 93.1785 & 11 & 0.000 \%\end{array}$

Seasonality present at the one percent level.

Moving Seasonality Test

$\begin{array}{ccccc} & \text { Sum of } & \text { Dgrs.of } & \text { Mean } & \\ \text { Squares } & \text { Freedom } & \text { Square } & \text { F-value } \\ \text { Between Years } & 0.9324 & 8 & 0.116546 & 10.566^{* *} \\ \text { Error } & 0.9706 & 88 & 0.011030 & \end{array}$

**Moving seasonality present at the one percent level.

Figura 2. Pruebas de Estacionalidad para el IPC Colombiano

En la Figura 3 se presenta la serie mensual de la TRM en Colombia, en ella se aprecia un comportamiento no estacionario, comprobado más adelante con pruebas de raíz unitaria.

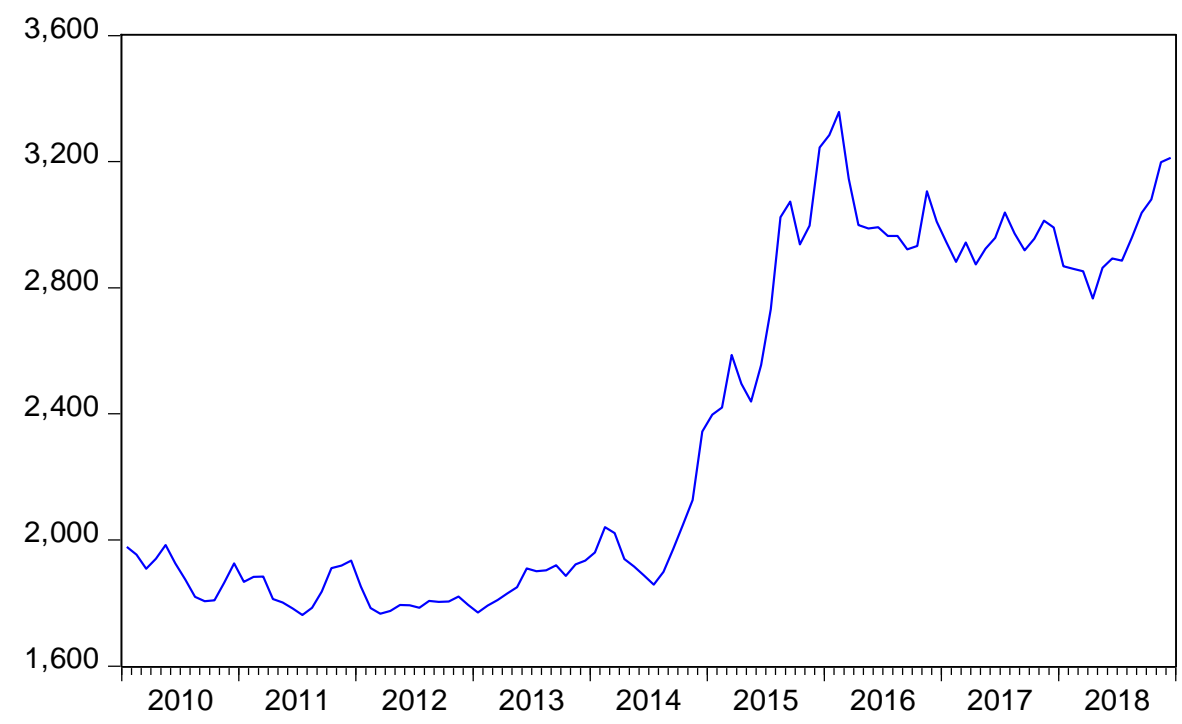

Figura 3. TRM mensual

Fuente: Banco de la República

En la Figura 4 se presenta la serie mensual del TCR en Colombia, en ella se aprecia un comportamiento no estacionario, comprobado más adelante con pruebas de raíz unitaria. 


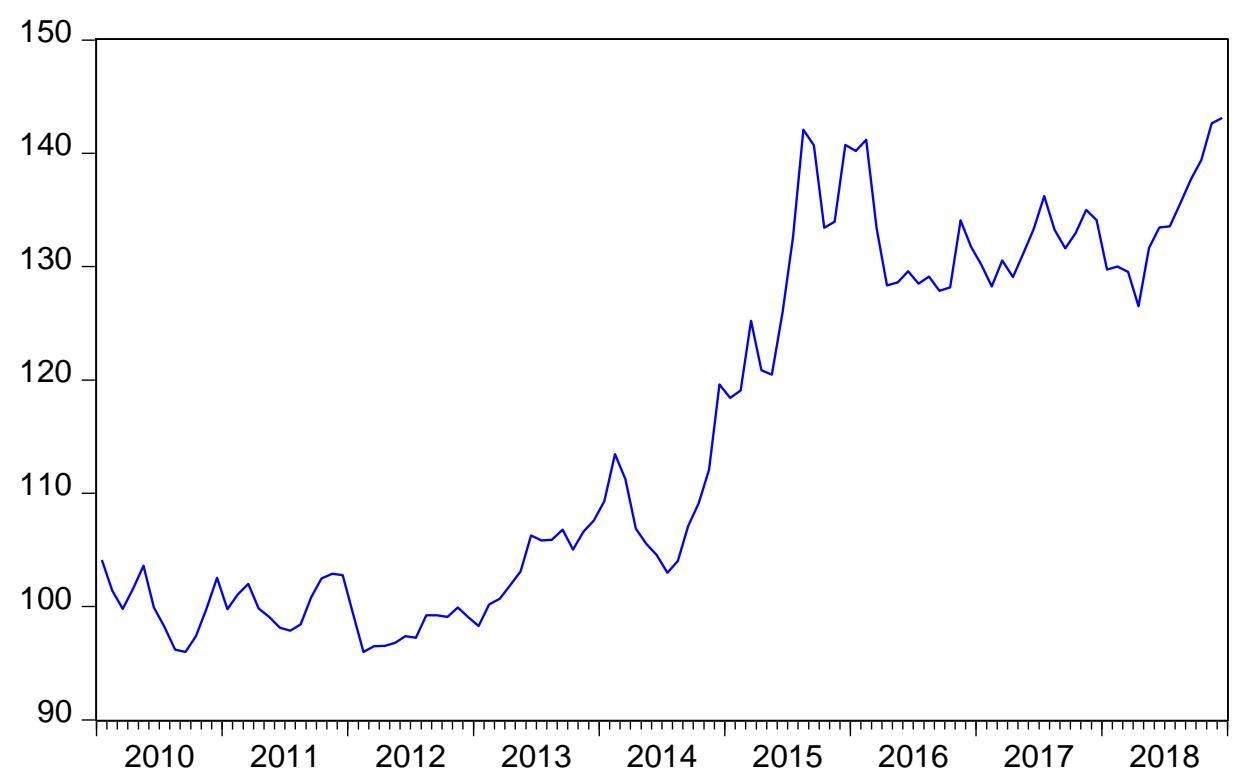

Figura 4. TCR mensual

Fuente: Banco de la Republica

Análisis de Integración (Pruebas D-F)

Para la utilización del modelo VEC, se requiere que las variables incorporadas al modelo sean estacionarias tengan orden de integración I(1), por lo cual se analizó el grado de integración. Para determinar si las series transformadas tienen raíces unitarias (verificar la estacionariedad), se utilizó la prueba de Dickey-Füller (D-F) implementada en el programa Eviews 10, además las series fueron ajustadas con la transformación logaritmo natural (denotada por la letra L que antecederá a la variable) y el operador diferencia (denotado por la letra D que antecederá a la variable). Para la variable LIPC_SA la estadística t fue de 0.906008 y para la variable DLIPC_SA la estadística t fue de 5.346049 , para la variable LTRM la estadística t fue de -0.404792 y para la variable DLTRM la estadística t fue de -7.606545 , para la variable LTCR la estadística t fue de -0.223917 y para la variable DLTCR la estadística t fue de -8.452601. Con los resultados anteriores se garantiza el orden de integración I(1) en todas las variables.

Especificación del modelo VEC para las variables DLIPC_SA - DLTRM

Dadas las pruebas de cointegración, se estimó el modelo de VEC para las variables DLIPC_SA - DLTRM, y para la elección del mejor modelo se utilizó el Criterio de BIC (BIC). Los resultados obtenidos se presentan en la Tabla 1.

\begin{tabular}{|c|c|c|c|c|}
\hline & $\mathrm{VEC}(1)$ & $\mathrm{VEC}(2)$ & $\mathrm{VEC}(3)$ & $\mathrm{VEC}(4)$ \\
\hline $\mathrm{BIC}$ & -14.09030 & -14.08731 & -14.19286 & -14.12546 \\
\hline
\end{tabular}


Tabla 1: Criterio de Información Bayesiano (AIC) para los posibles modelos VEC.

En la figura 5 se presenta el ajuste del modelo VEC escogido empleando el programa Eviews 10.

Vector Error Correction Estimates

Sample (adjusted): 2010M06 2018M12

Included observations: 103 after adjustments

Standard errors in ( ) \& t-statistics in [ ]

\begin{tabular}{|c|c|c|}
\hline Cointegrating Eq: & CointEq1 & \\
\hline DLIPC_SA(-1) & 1.000000 & \\
\hline $\operatorname{DLTRM}(-1)$ & $\begin{array}{r}-0.280878 \\
(0.05133) \\
{[-5.47203]}\end{array}$ & \\
\hline $\mathrm{C}$ & -0.001792 & \\
\hline Error Correction: & $\begin{array}{c}\mathrm{D}\left(\mathrm{DLIPC}_{-}\right. \\
\mathrm{SA})\end{array}$ & $\mathrm{D}(\mathrm{DLTRM})$ \\
\hline CointEq1 & $\begin{array}{r}-0.107836 \\
(0.03527) \\
{[-3.05764]}\end{array}$ & $\begin{array}{r}2.688692 \\
(0.65769) \\
{[4.08805]}\end{array}$ \\
\hline $\mathrm{D}($ DLIPC_SA $(-1))$ & $\begin{array}{r}-0.434031 \\
(0.08918) \\
{[-4.86715]}\end{array}$ & $\begin{array}{r}-1.150254 \\
(1.66301) \\
{[-0.69167]}\end{array}$ \\
\hline D(DLIPC_SA(-2)) & $\begin{array}{r}-0.324898 \\
(0.09500) \\
{[-3.41998]}\end{array}$ & $\begin{array}{r}-3.580019 \\
(1.77163) \\
{[-2.02075]}\end{array}$ \\
\hline $\mathrm{D}($ DLIPC_SA $(-3))$ & $\begin{array}{r}-0.300649 \\
(0.08961) \\
{[-3.35501]}\end{array}$ & $\begin{array}{r}-2.602208 \\
(1.67115) \\
{[-1.55714]}\end{array}$ \\
\hline
\end{tabular}




\begin{tabular}{crr}
$\mathrm{D}(\operatorname{DLTRM}(-1))$ & -0.010044 & 0.124451 \\
& $(0.00912)$ & $(0.17016)$ \\
& {$[-1.10070]$} & {$[0.73136]$} \\
& & \\
$\mathrm{D}(\operatorname{DLTRM}(-2))$ & -0.004791 & -0.033159 \\
& $(0.00715)$ & $(0.13338)$ \\
& {$[-0.66978]$} & {$[-0.24860]$} \\
$\mathrm{D}(\mathrm{DLTRM}(-3))$ & 0.002152 & 0.102931 \\
& $(0.00578)$ & $(0.10771)$ \\
$\mathrm{C}$ & {$[0.37251]$} & {$[0.95563]$} \\
& $9.93 \mathrm{E}-06$ & -0.000219 \\
& $(0.00015)$ & $(0.00283)$ \\
& {$[0.06536]$} & {$[-0.07717]$} \\
\hline \hline
\end{tabular}

Figura 5. Ajuste modelo VEC(3) para las variables DLIPC_SA - DLTRM

Se aprecia el vector de corrección o vector de cointegración $\beta^{\prime}=(1,-0.280878$, $0.001792)$. En él, se evidencia que los coeficientes fueron significativos al $5 \%$, se ha normalizado para que el primer coeficiente sea igual a uno. Dicho vector presenta el equilibrio de largo plazo del sistema el cual se puede representar como DLIPC_SA $A_{t}$ $0.280878 D L T R M_{t}=0$, de donde se deduce que $D L I P C_{-} S A_{t}=0.280878 D L T R M_{t}$ y por lo tanto un incremento en la variable DLTRM, generará un incremento en la variable DLIPC_SA en el largo plazo. Esta conclusión se validará con la función impulso respuesta. 


\section{Response of DLIPC_SA to DLTRM Innovation using Cholesky (d.f. adjusted) Factors}

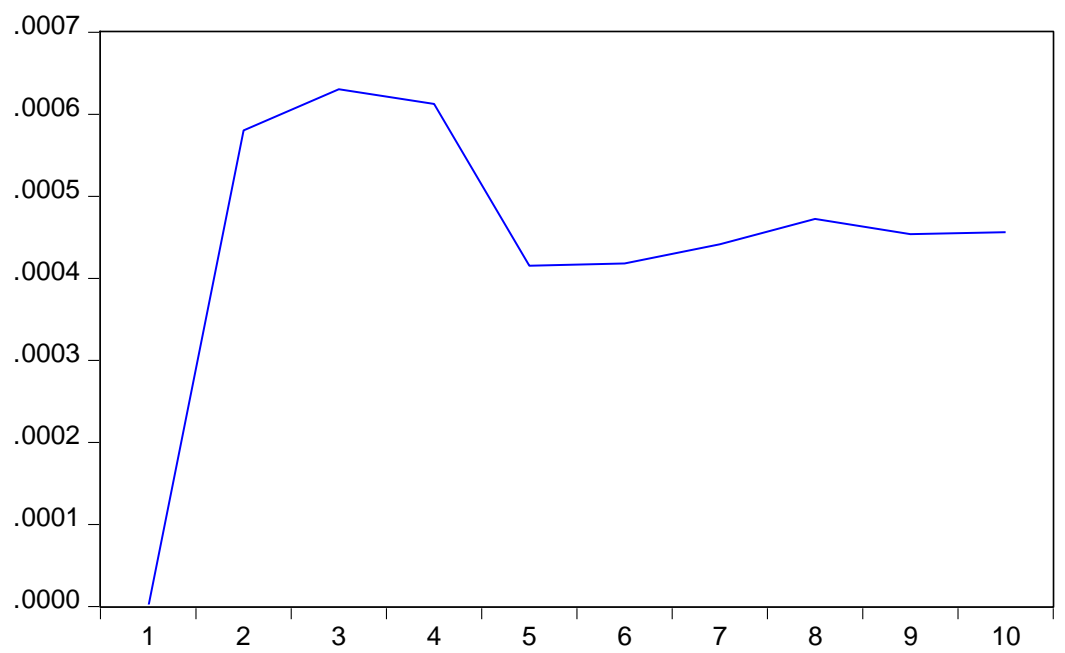

Figura 6. Función Impulso-Respuesta para las variables DLIPC_SA-DLTRM

La Figura 6 evidencia la función impulso respuesta de la variable DLIPC_SA ante un choque de la variable DLTRM, se presenta un efecto positivo el cual se mantiene en el largo plazo. Una disminución en la TRM, implica un efecto valor directo generador de un incremento en el precio de los insumos y bienes finales importados en términos de la moneda local. El sector empresarial, con el fin de impedir modificaciones en sus márgenes de ganancia o producir pérdidas en el largo plazo, podría transmitir parte del aumento en los costos a los consumidores finales, originando presiones inflacionarias en la economía.

Especificación del modelo VEC para las variables DLIPC_SA - DLTCR Dadas las pruebas de cointegración, se estimó el modelo de VEC para las variables DLIPC_SA - DLTCR, además para la elección del mejor modelo se utilizó el Criterio de Akaike (AIC). Los resultados obtenidos se presentan en la Tabla 2.

\begin{tabular}{|c|c|c|c|c|}
\hline & $\mathrm{VEC}(1)$ & $\mathrm{VEC}(2)$ & $\mathrm{VEC}(3)$ & $\mathrm{VEC}(4)$ \\
\hline $\mathrm{BIC}$ & -14.53925 & -14.53437 & -14.65432 & -14.61009 \\
\hline
\end{tabular}

Tabla 2: Criterio de Información Bayesiano (BIC) para los posibles modelos VEC.

En la figura 7 se presenta el ajuste del modelo VEC escogido empleando el programa Eviews 10.

Vector Error Correction Estimates 
Sample (adjusted): 2010M06 2018M12

Included observations: 103 after adjustments

Standard errors in ( ) \& t-statistics in [ ]

\begin{tabular}{|c|c|c|}
\hline Cointegrating Eq: & CointEq1 & \\
\hline DLIPC_SA(-1) & 1.000000 & \\
\hline $\operatorname{DLTCR}(-1)$ & $\begin{array}{r}-1.687946 \\
(0.28287) \\
{[-5.96724]}\end{array}$ & \\
\hline $\mathrm{C}$ & 0.002410 & \\
\hline Error Correction: & $\begin{array}{c}\mathrm{D}\left(\mathrm{DLIPC}_{-}\right. \\
\mathrm{SA})\end{array}$ & $\mathrm{D}(\mathrm{DLTCR})$ \\
\hline CointEq1 & $\begin{array}{r}-0.024694 \\
(0.00828) \\
{[-2.98220]}\end{array}$ & $\begin{array}{r}0.599155 \\
(0.11881) \\
{[5.04301]}\end{array}$ \\
\hline $\mathrm{D}($ DLIPC_SA $(-1))$ & $\begin{array}{r}-0.483828 \\
(0.09224) \\
{[-5.24543]}\end{array}$ & $\begin{array}{r}0.125682 \\
(1.32344) \\
{[0.09497]}\end{array}$ \\
\hline $\mathrm{D}($ DLIPC_SA $(-2))$ & $\begin{array}{r}-0.355910 \\
(0.09965) \\
{[-3.57147]}\end{array}$ & $\begin{array}{r}-1.989293 \\
(1.42984) \\
{[-1.39127]}\end{array}$ \\
\hline $\mathrm{D}($ DLIPC_SA $(-3))$ & $\begin{array}{r}-0.318142 \\
(0.09313) \\
{[-3.41616]}\end{array}$ & $\begin{array}{r}-1.271084 \\
(1.33622) \\
{[-0.95126]}\end{array}$ \\
\hline $\mathrm{D}(\operatorname{DLTCR}(-1))$ & $\begin{array}{r}-0.018168 \\
(0.01236) \\
{[-1.46953]}\end{array}$ & $\begin{array}{r}0.266712 \\
(0.17738) \\
{[1.50360]}\end{array}$ \\
\hline $\mathrm{D}(\operatorname{DLTCR}(-2))$ & $\begin{array}{r}-0.010224 \\
(0.00935) \\
{[-1.09289]}\end{array}$ & $\begin{array}{r}0.030742 \\
(0.13422) \\
{[0.22904]}\end{array}$ \\
\hline D(DLTCR $(-3))$ & 0.001846 & 0.156694 \\
\hline
\end{tabular}

Comunicaciones en Estadística Junio 2019, Vol. 12, No. 1 
$(0.00723) \quad(0.10373)$

[0.25539] [ 1.51061$]$

C $\quad 8.94 \mathrm{E}-06 \quad-0.000220$

$(0.00015) \quad(0.00221)$

$[0.05797] \quad[-0.09937]$

Figura 7. Ajuste modelo VEC(3) para las variables DLIPC_SA - DLTCR

Se aprecia el vector de corrección o vector de cointegración $\beta "=(1,-1.687946$, 0.002410). En él se evidencia que los coeficientes fueron significativos al 5\%. Además, se ha normalizado para que el primer coeficiente sea igual a uno. Dicho vector presenta el equilibrio de largo plazo del sistema el cual se puede representar como DLIPC_SA $A_{t}$ 1.687946 LITCR $_{t}=0$, de donde se deduce que DLIPC_SA $A_{t}=1.687946 D L T R M_{t}$ y por lo tanto un incremento en la variable DLTCR, generará un incremento en la variable DLIPC_SA en el largo plazo. Esta conclusión se validará con la función impulso respuesta.

\section{Response of DLIPC_SA to DLTCR Innovation using Cholesky (d.f. adjusted) Factors}

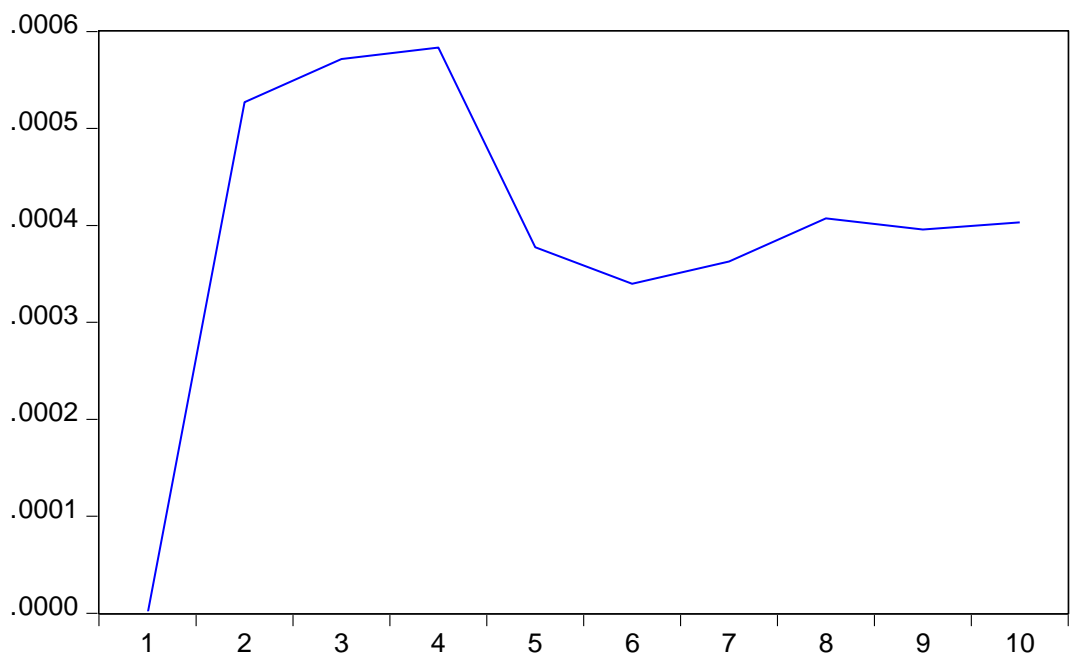

Figura 8. Función Impulso-Respuesta para las variables DLIPC_SA-DLTCR

La Figura 6 expone la función impulso respuesta de la variable DLIPC_SA ante un choque de la variable DLTCR, se presenta un efecto positivo el cual se mantiene en el largo plazo. Un aumento en el TCR, manifiesta un incremento en la competitividad de los precios internos frente a los internacionales, suponiendo un alza en la demanda por la producción nacional, generador de presiones inflacionarias en la economía. Las 
ventajas que representa su depreciación al sector exportador, podrían verse mitigadas de no efectuarse un debido control en el efecto alcista que este causa sobre los precios internos en el largo plazo, constante a partir de 4 meses posterior al choque inicial.

Diagnóstico Econométrico del VEC

La flexibilidad del VEC respecto a otros modelos econométricos representa una de sus principales ventajas, debido a la endogeneidad de las variables que se incluyan en el análisis, por lo cual no analiza los coeficientes de regresión estimados ni sus significancias estadísticas, tampoco se tiene en cuenta la bondad de ajuste (R2 ajustado) de las ecuaciones individuales. Sin embargo, se debe verificar la ausencia de correlación serial de los residuos de las ecuaciones individuales del modelo y la distribución normal multivariada de estos.

\section{A utocorrelación}

Los correlogramas y pruebas Portmanteau de los residuos de las dos ecuaciones del VEC, y las correlaciones presentadas por las combinaciones de variables contemporáneas y de las variables rezagadas del modelo no evidencian problemas de autocorrelación. (Ver Anexo 2.)

\section{Normalidad}

Para todos los modelos VEC ajustados, los residuos de las ecuaciones no tienen una distribución normal. No obstante, Fernández-Corugedo (2003) argumenta que por encima de la normalidad multivariada, el cumplimiento del modelo con la prueba de errores no autocorrelacionados, presenta mayor relevancia.

\section{Conclusiones}

A partir del grado de estacionariedad I(1) de las variables de estudio y la cointegración de las mismas, se utilizó el modelo VEC (Vector de Corrección de Errores), dado el cumplimiento de los supuestos iníciales. Con base en la revisión del problema inflacionario, evaluado a partir del fenómeno de inflación importada, que manifiesta las repercusiones de variables externas sobre el nivel de precios interno, se evidencian los impactos de las variables TRM e TCR en la inflación colombiana.

El presente estudio, a partir las relaciones de cointegración en el largo plazo de las variables Tasa Representativa de Mercado (TRM) e Índice de Tasa de Cambio Real (TCR) con el Índice de Precios al Consumidor (IPC) para Colombia, comprueba los impactos positivos que serán generados en el IPC, por el choque del aumento de una desviación estándar en las variables TRM e TCR, en un periodo de 24 meses, sustentado en las Funciones Impulso Respuesta (FIR). 
Se demuestra un coeficiente de traspaso positivo tendiente a mantenerse en el largo plazo, actuando en detrimento del poder adquisitivo de la moneda nacional, saldos reales y la balanza comercial, bajo la ausencia de políticas económicas reguladoras. El anterior análisis se realiza en aras de exponer la preponderancia de la implementación del Índice de Inflación Importada para la economía colombiana, dadas las múltiples vías de acceso que esta encuentra sobre el nivel de precios nacional, expuestas a lo largo de este documento.

\section{ANEXOS}

Anexo1. Pruebas de cointegración.

\begin{tabular}{|c|c|c|c|c|}
\hline \multicolumn{5}{|c|}{$\begin{array}{l}\text { Sample (adjusted): } 2010 \mathrm{M} 04 \text { 2018M12 } \\
\text { Included observations: } 105 \text { after adjustments } \\
\text { Trend assumption: Linear deterministic trend } \\
\text { Series: DLIPC_SA DLTRM } \\
\text { Lags interval (in first differences): } 1 \text { to } 1\end{array}$} \\
\hline \multicolumn{5}{|c|}{ Unrestricted Cointegration Rank Test (Trace) } \\
\hline $\begin{array}{l}\text { Hypothesized } \\
\text { No. of } C E(s)\end{array}$ & Eigenvalue & $\begin{array}{l}\text { Trace } \\
\text { Statistic }\end{array}$ & $\begin{array}{c}0.05 \\
\text { Critical Value }\end{array}$ & Prob.** \\
\hline $\begin{array}{c}\text { None * } \\
\text { At most } 1 \text { * }\end{array}$ & $\begin{array}{l}0.374785 \\
0.147596\end{array}$ & $\begin{array}{l}66.08227 \\
16.76800\end{array}$ & $\begin{array}{l}15.49471 \\
3.841466\end{array}$ & $\begin{array}{l}0.0000 \\
0.0000\end{array}$ \\
\hline \multicolumn{5}{|c|}{$\begin{array}{l}\text { Trace test indicates } 2 \text { cointegrating eqn(s) at the } 0.05 \text { level } \\
\text { * denotes rejection of the hypothesis at the } 0.05 \text { level } \\
\text { **MacKinnon-Haug-Michelis (1999) p-values }\end{array}$} \\
\hline \multicolumn{5}{|c|}{ Unrestricted Cointegration Rank Test (Maximum Eigenvalue) } \\
\hline $\begin{array}{l}\text { Hypothesized } \\
\text { No. of } C E(s)\end{array}$ & Eigenvalue & $\begin{array}{l}\text { Max-Eigen } \\
\text { Statistic }\end{array}$ & $\begin{array}{c}0.05 \\
\text { Critical Value }\end{array}$ & Prob.** \\
\hline None * & 0.374785 & 49.31427 & 14.26460 & 0.0000 \\
\hline At most 1 * & 0.147596 & 16.76800 & 3.841466 & 0.0000 \\
\hline
\end{tabular}

\begin{tabular}{|c|c|c|c|c|}
\hline \multicolumn{5}{|c|}{$\begin{array}{l}\text { Sample (adjusted): } 2010 \mathrm{M} 042018 \mathrm{M} 12 \\
\text { Included observations: } 105 \text { after adjustments } \\
\text { Trend assumption: Linear deterministic trend } \\
\text { Series: DLIPC_SA DLTCR } \\
\text { Lags interval (in first differences): } 1 \text { to } 1\end{array}$} \\
\hline \multicolumn{5}{|c|}{ Unrestricted Cointegration Rank Test (Trace) } \\
\hline $\begin{array}{l}\text { Hypothesized } \\
\text { No. of } C E(s)\end{array}$ & Eigenvalue & $\begin{array}{l}\text { Trace } \\
\text { Statistic }\end{array}$ & $\begin{array}{c}0.05 \\
\text { Critical Value }\end{array}$ & Prob.** \\
\hline $\begin{array}{c}\text { None * } \\
\text { At most } 1 \text { * }\end{array}$ & $\begin{array}{l}0.425845 \\
0.144439\end{array}$ & $\begin{array}{l}74.63954 \\
16.37974\end{array}$ & $\begin{array}{l}15.49471 \\
3.841466\end{array}$ & $\begin{array}{l}0.0000 \\
0.0001\end{array}$ \\
\hline \multicolumn{5}{|c|}{$\begin{array}{l}\text { Trace test indicates } 2 \text { cointegrating eqn(s) at the } 0.05 \text { level } \\
{ }^{*} \text { denotes rejection of the hypothesis at the } 0.05 \text { level } \\
\text { **MacKinnon-Haug-Michelis (1999) p-values }\end{array}$} \\
\hline \multicolumn{5}{|c|}{ Unrestricted Cointegration Rank Test (Maximum Eigenvalue) } \\
\hline $\begin{array}{l}\text { Hypothesized } \\
\text { No. of } C E(s)\end{array}$ & Eigenvalue & $\begin{array}{l}\text { Max-Eigen } \\
\text { Statistic }\end{array}$ & $\begin{array}{c}0.05 \\
\text { Critical Value }\end{array}$ & Prob.** \\
\hline None * & 0.425845 & 58.25981 & 14.26460 & 0.0000 \\
\hline At most 1 * & 0.144439 & 16.37974 & 3.841466 & 0.0001 \\
\hline
\end{tabular}

Anexo 2. Autocorrelación

\begin{tabular}{|c|c|c|c|c|c|}
\hline Lags & Q-Stat & Prob. $^{*}$ & Adj Q-Stat & Prob.* & df \\
\hline 1 & 0.067740 & - & 0.068404 & - & - \\
\hline 2 & 0.194339 & - & 0.197510 & - & - \\
\hline 3 & 0.669625 & - & 0.687055 & $\ldots$ & - \\
\hline 4 & 3.905833 & 0.6894 & 4.054018 & 0.6694 & 6 \\
\hline 5 & 9.225047 & 0.5109 & 9.644621 & 0.4722 & 10 \\
\hline 6 & 10.96377 & 0.6889 & 11.49089 & 0.6471 & 14 \\
\hline 7 & 17.85297 & 0.4654 & 18.88244 & 0.3991 & 18 \\
\hline 8 & 21.22826 & 0.5067 & 22.54196 & 0.4280 & 22 \\
\hline 9 & 23.43442 & 0.6083 & 24.95934 & 0.5213 & 26 \\
\hline 10 & 25.71585 & 0.6896 & 27.48609 & 0.5976 & 30 \\
\hline 11 & 31.90706 & 0.5706 & 34.41755 & 0.4478 & 34 \\
\hline 12 & 33.57374 & 0.6742 & 36.30402 & 0.5480 & 38 \\
\hline
\end{tabular}

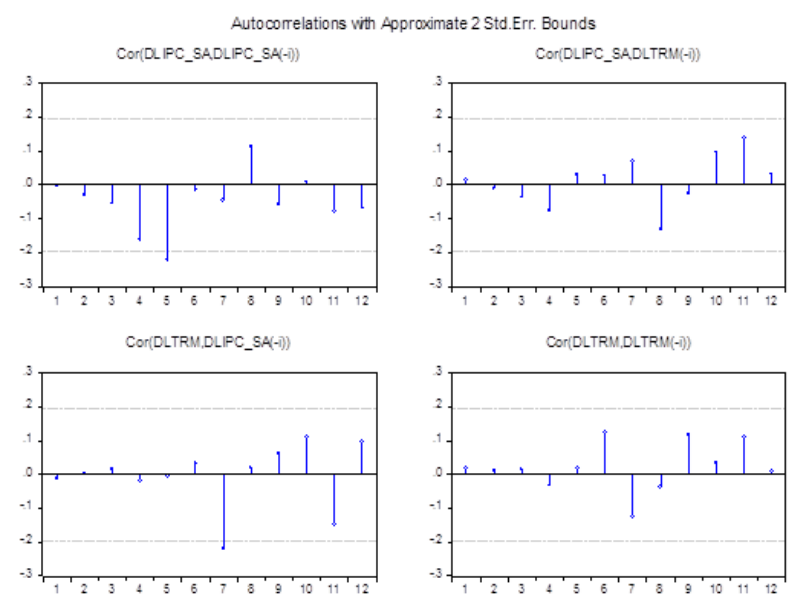

Comunicaciones en Estadística Junio 2019, Vol. 12, No. 1 


\begin{tabular}{|c|c|c|c|c|c|}
\hline Lags & Q-Stat & Prob. ${ }^{*}$ & Adj Q-Stat & Prob.* & df \\
\hline 1 & 0.199580 & - & 0.201536 & - & - \\
\hline 2 & 0.373027 & - & 0.378418 & - & - \\
\hline 3 & 0.800974 & - & 0.819204 & - & - \\
\hline 4 & 4.070913 & 0.6671 & 4.221261 & 0.6468 & 6 \\
\hline 5 & 8.699992 & 0.5608 & 9.086518 & 0.5239 & 10 \\
\hline 6 & 9.792915 & 0.7772 & 10.24704 & 0.7439 & 14 \\
\hline 7 & 16.06164 & 0.5882 & 16.97287 & 0.5250 & 18 \\
\hline 8 & 18.97417 & 0.6469 & 20.13066 & 0.5749 & 22 \\
\hline 9 & 20.60242 & 0.7622 & 21.91481 & 0.6933 & 26 \\
\hline 10 & 24.39596 & 0.7538 & 26.11625 & 0.6692 & 30 \\
\hline 11 & 29.03657 & 0.7095 & 31.31172 & 0.6001 & 34 \\
\hline 12 & 32.18512 & 0.7346 & 34.87546 & 0.6147 & 38 \\
\hline
\end{tabular}

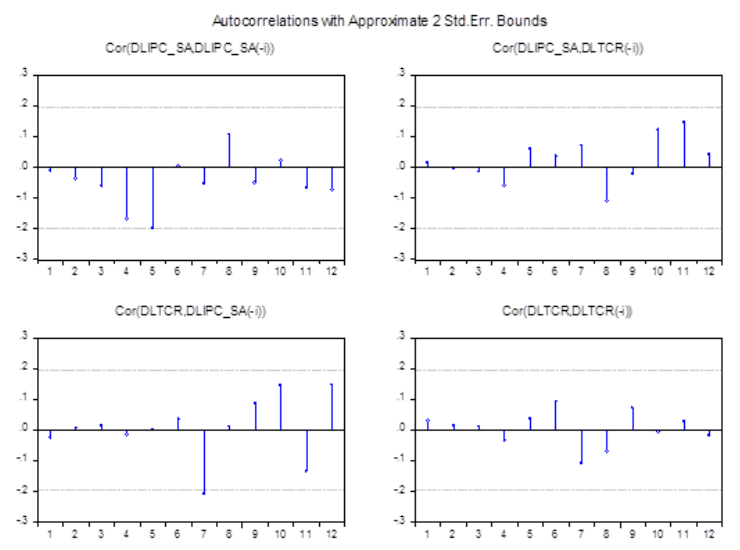

Recibido: 2019-02-24

Aceptado: 2019-06-17

\section{Referencias}

Banco de la República (Banco central de Colombia). Recuperado de http://www.banrep.gov.co/es/ipc.

Cárdenas, M. (2013). Introducción a la economía colombiana (1st ed.). Bogotá, D.C.: Alfaomega.

Engle, R \& Granger, C. (1987). Co-Integration and error correction: representation, estimation, and testing. Econometrica, 55(2), 251-76.

Fernandez-Corugedo, E. (2003). Exercise on unit roots (incluiding structural breaks), estimating a VECM and the implications of the VECM, Technical report. Bank of England.

Frenkel, R. (2008). Las políticas monetaria y fiscal en un régimen de tipo de cambio competitivo. Economia e Sociedade, 17(spe), 611-628.

Frisch, H. (1977). Inflation theory 1968-1975: A "second generation" survey, Journal of Economic Literature, 15(4), 1289-1317. 
Gallardo, M. \& Rubio, H (2009). Diagnóstico de Estacionalidad con X12-ARIMA. Serie Estudios Económicos Estadísticos (76). Banco Central de Chile.

González, A., Rincón, H. \& Rodríguez, N. (2008). La transmisión de los choques a la tasa de cambio sobre la inflación de los bienes importados en presencia de asimetrías. Borradores de Economía (532) Bogotá, Colombia.

Johansen, S. \& Juselius, K. (1990), Maximum likelihood estimation and inference on cointegration - with applications to the demand for money. Oxford Bulletin of Economics and Statistics, 52, 169-210.

Kikut, A. \& Ocampo, A. (2005). Ajuste estacional de series económicas con tramo/seatsy census x12-arima, Nota técnica NT-01-2005. Banco Central de Costa Rica.

Krugman, P. \& Obstfeld, M. (1994). Economía internacional: Teoría y política, Madrid, España: Pearson.

Lettau, M. \& Ludvigson, S. (2001). Consumption Aggregate Wealth, and Expected Stock Returns, The Jorurnal of finance, 56(3), 815 -848.

Mundell, R. (1963). Capital Mobility and Stabilization Policy under Fixed and Flexible Exchange Rates. The Canadian Journal of Economics and Political Science, 29(4), 475-485.

Rendón, H. \& Ramírez, A. (2005), Condición Marshall-Lerner: una aproximación al caso colombiano, 1980-2001. Ecos de Economía, 9(20), 29-58.

Rincón, H., Lozano, I. \& Ramos, J. (2008) Rentas petroleras, subsidios e impuestos a los combustibles en Colombia: ¿Qué ocurrió durante el choque reciente de precios?, Borradores de Economía (541).

Rincón, H. (2000). Devaluación y precios agregados en Colombia, 1980- 1998, Desarrollo y Sociedad, 46, 109-144.

Rodríguez, H. (2011), Estudio del fenómeno de inflación importada vía precios del petróleo y su aplicación al caso colombiano mediante el uso de modelos VAR para el periodo 2000-2009. Estudios Gerenciales, 27:121, pp. 79-98. https://doi.org/10.1016/S0123-5923(11)70182-6

Ross, S., Westerfield, R., Jaffe, J. \& Jordan, B. (2010). Fundamentos de Finanzas Corporativas. México: Editorial Mc. Graw Hill. 
Solé, R. (2012), Efectos de la inflación y la devaluación en la evaluación de flujos de inversión. Ciencias Económicas, 30, 279.

Urrutia, M. (2002). Una visión alternativa: la política monetaria y cambiaria en la última década, Borradores de Economía (207).

Urrutia, M. (2004). La tasa de cambio y la política monetaria de inflación objetivo [Nota editorial]. Revista del Banco de la República, 78(916), 5-13.

Wei, W. (2006), Time Series Analysis: Univariate and Multivariate Methods, Boston, Estados Unidos: Addison-Wesley. 